\title{
RIBBON-MOVES FOR 2-KNOTS WITH 1-HANDLES ATTACHED AND KHOVANOV-JACOBSSON NUMBERS
}

\author{
J. SCOTT CARTER, MASAHICO SAITO, AND SHIN SATOH \\ (Communicated by Ronald A. Fintushel)
}

\begin{abstract}
We prove that a crossing change along a double point circle on a 2-knot is realized by ribbon-moves for a knotted torus obtained from the 2-knot by attaching a 1-handle. It follows that any 2-knots for which the crossing change is an unknotting operation, such as ribbon 2-knots and twistspun knots, have trivial Khovanov-Jacobsson number.
\end{abstract}

A surface-knot or -link is a closed surface embedded in 4-space $\mathbb{R}^{4}$ locally flatly. Throughout this note, we always assume that all surface-knots are oriented. A ribbon-move (cf. [10]) is a local operation for (a diagram of) a surface-knot as shown in Figure 1. We say that surface-knots $F$ and $F^{\prime}$ are ribbon-move equivalent, denoted by $F \sim F^{\prime}$, if $F^{\prime}$ is obtained from $F$ by a finite sequence of ribbon-moves.

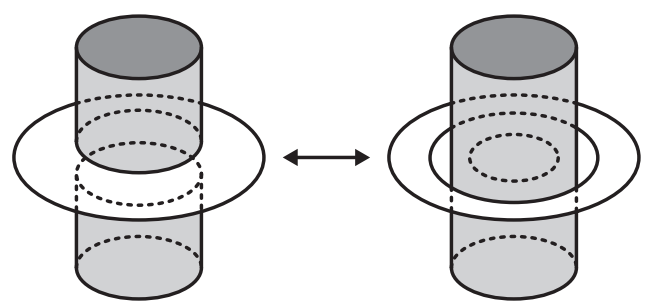

Figure 1.

The ribbon-move is a special case of the crossing change: Assume that a surfaceknot $F$ has a double point circle $L$ in a diagram such that (i) $L$ has no selfintersection, and (ii) at every triple point on $L$, the sheet transverse to $L$ is either top or bottom (not middle). The condition (i) means that $L$ does not go through the same triple point twice. When $L$ satisfies these conditions, we can perform a crossing change along $L$ by exchanging the roles of over- and under-sheets as indicated in Figure 2 (cf. [16]). See [4] for details on diagrams of surface-knots.

Received by the editors October 19, 2004 and, in revised form, April 14, 2005.

2000 Mathematics Subject Classification. Primary 57Q45; Secondary 57Q35.

Key words and phrases. Khovanov homology, 2-knot, ribbon-move, twist-spun knot, crossing change.

The first author was supported in part by NSF Grant DMS \#0301095.

The second author was supported in part by NSF Grant DMS \#0301089.

The third author was supported in part by JSPS Postdoctoral Fellowships for Research Abroad. 


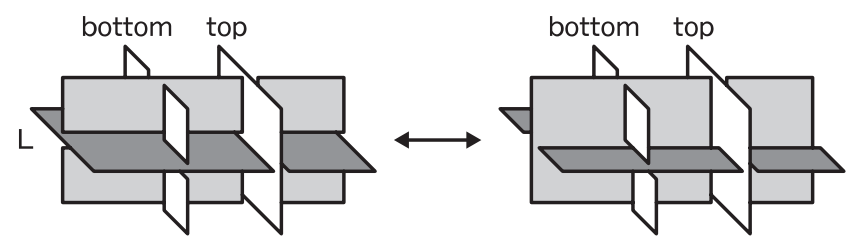

FIGURE 2.

For a 2-knot $K$ (a knotted sphere in $\mathbb{R}^{4}$ ), a crossing change is not necessarily realized by ribbon-moves; indeed, a ribbon-move does not change the Farber-Levine pairing of $K$, but a crossing change might (cf. [10]). On the other hand, when we consider the $\mathbb{T}^{2}$-knot (knotted torus in $\mathbb{R}^{4}$ ) $K+h$ obtained from $K$ by attaching a 1-handle $h$ on $K$, we obtain the following.

Theorem 1. Let $K$ and $K^{\prime}$ be 2-knots such that $K^{\prime}$ is obtained from $K$ by a crossing change. Then for any 1-handles $h$ and $h^{\prime}$ on $K$ and $K^{\prime}$, respectively, the $\mathbb{T}^{2}$-knot $K+h$ is ribbon-move equivalent to $K^{\prime}+h^{\prime}$.

Proof. Along the double point circle $L$ for which we perform the crossing change, there is a neighborhood $N$ identified with $\left(B^{3}, t\right) \times S^{1}$, where $\left(B^{3}, t\right)$ is a tangle with two strings as shown on the left of Figure 3 . In the figure, the orientations of tangles are induced from that of $K$, and all bands are attached in an orientationcompatible manner. For an interval $I$ in $S^{1}$, we take a 1-handle $h_{1}=b_{1} \times I$ on $K$, where $b_{1}$ is a band as indicated in the figure.

We observe that $K+h_{1}$ is ambient isotopic to $\left(K^{\prime} \cup T\right)+h_{2}$ (cf. [12]), where $T=m \times S^{1}$ is a $\mathbb{T}^{2}$-knot linking with $K^{\prime}$, and the 1-handle $h_{2}=b_{2} \times I$ connects between $K^{\prime}$ and $T$. See the center of Figure 3 .

Consider a 1-handle $h_{3}=b_{3} \times I$ on $K^{\prime} \cup T$. Since both $h_{2}$ and $h_{3}$ connect between $K^{\prime}$ and $T$, the $\mathbb{T}^{2}$-knot $\left(K^{\prime} \cup T\right)+h_{2}$ is ribbon-move equivalent to $\left(K^{\prime} \cup T\right)+h_{3}$.

Finally we see that $\left(K^{\prime} \cup T\right)+h_{3}$ is ambient isotopic to $K^{\prime}+h_{4}$, where $h_{4}=b_{4} \times I$ is the 1-handle on $K^{\prime}$ as shown on the right of the figure. Thus we obtain

$$
K+h \sim K+h_{1}=\left(K^{\prime} \cup T\right)+h_{2} \sim\left(K^{\prime} \cup T\right)+h_{3}=K^{\prime}+h_{4} \sim K^{\prime}+h^{\prime} .
$$

This completes the proof.
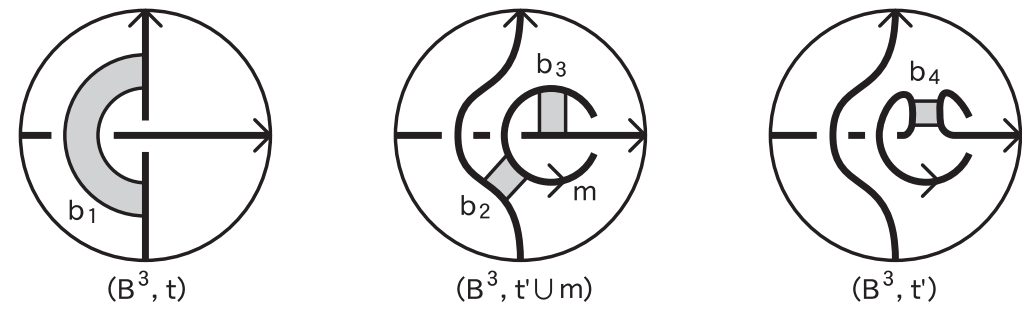

FiguRE 3.

We say that the crossing change is an unknotting operation for a surface-knot $F$ if the trivial surface-knot is obtained from $F$ by a finite sequence of crossing changes. It is still unknown whether the crossing change is an unknotting operation for any surface-knot. 
Khovanov [8] introduced a categorification of the Jones polynomial, that is, a chain complex for a given classical knot diagram such that its graded Euler characteristic is the Jones polynomial. Khovanov [9] and Jacobsson [5] proved that it defines an invariant for cobordisms (relative to boundary diagrams). Specifically, a cobordism between two knot diagrams gives rise to a chain map (we call it a Khovanov-Jacobsson homomorphism) between corresponding chain complexes that is invariant under equivalence of cobordisms of diagrams. See also 2]. In particular, a diagram of a $\mathbb{T}^{2}$-knot is a cobordism between empty diagrams, giving rise to a homomorphism $\mathbb{Z} \rightarrow \mathbb{Z}$ defined up to sign, a multiplication by a constant. We call this constant the Khovanov-Jacobsson number.

Theorem 2. Let $K$ be a 2-knot for which a crossing change is an unknotting operation. Then for any 1 -handle $h$ on $K$, the $\mathbb{T}^{2}$-knot $K+h$ has trivial KhovanovJacobsson number.

Proof. Let $K_{0}$ be the trivial 2-knot and $h_{0}$ the trivial 1-handle on $K_{0}$. By assumption and Theorem 1 , the $\mathbb{T}^{2}$-knot $K+h$ is ribbon-move equivalent to $K_{0}+h_{0}$, which is the trivial $\mathbb{T}^{2}$-knot.

Consider two movies as shown in Figure 4, It is seen from the definitions [2, 5] that the corresponding Khovanov-Jacobsson homomorphisms $H^{*}(\mid \bigcirc) \rightarrow H^{*}(\bigcirc \mid)$ are the same for these movies. This implies that a ribbon-move does not change the Khovanov-Jacobsson number. Hence the $\mathbb{T}^{2}$-knot $K+h$ has the same number as that of the trivial $\mathbb{T}^{2}$-knot $K_{0}+h_{0}$.

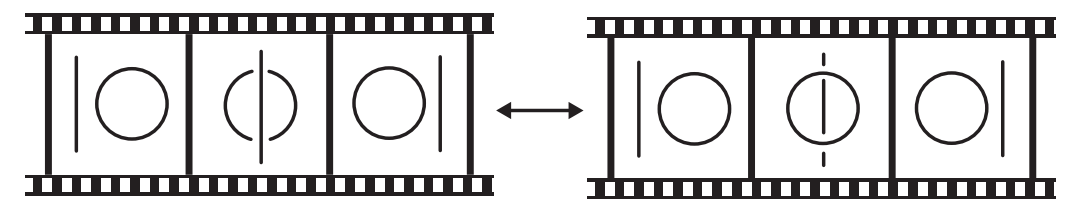

FiguRE 4.

By Theorem 2, if there is a 2-knot $K$ such that the Khovanov-Jacobsson number of $K+h$ is non-trivial, then the crossing change is not an unknotting operation for $K$. However, we have no such examples at present.

Corollary 3. Let $K$ be a ribbon 2-knot or twist-spun knot. Then for any 1-handle $h$ on $K$, the $\mathbb{T}^{2}$-knot $K+h$ has trivial Khovanov-Jacobsson number.

Proof. This follows from Theorem 2 and the fact that the crossing change is an unknotting operation for every ribbon 2-knot or twist-spun knot (cf. [1, 11]).

We say that a surface-knot is pseudo-ribbon [7] if it has a diagram without triple points. The notions of ribbon and pseudo-ribbon 2-knots are the same [15] (see also 6]). On the other hand, for $\mathbb{T}^{2}$-knots, they are not coincident in the sense that the family of pseudo-ribbon $\mathbb{T}^{2}$-knots properly contains that of ribbon $\mathbb{T}^{2}$-knots.

Proposition 4. Any pseudo-ribbon $\mathbb{T}^{2}$-knot has the trivial Khovanov-Jacobsson number. 
Proof. By the results of Teragaito [14] and Shima [13], every pseudo-ribbon $\mathbb{T}^{2}$-knot $T$ is (i) a ribbon $\mathbb{T}^{2}$-knot, or (ii) a $\mathbb{T}^{2}$-knot obtained from a split union of a Boyle's turned $\mathbb{T}^{2}$-knot $T^{\prime}\left[3\right.$ and a trivial 2-link $U=U_{1} \cup U_{2} \cup \cdots \cup U_{n}$ by surgery along 1-handles $h_{1}, h_{2}, \ldots, h_{n}$ for some $n \geq 0$, where each $h_{i}$ connects between $T^{\prime}$ and $h_{i}$ $(i=1,2, \ldots, n)$.

For the case (i), there is a ribbon 2-knot $K$ and a 1-handle $h$ such that $T=K+h$. Hence the conclusion follows from Corollary 3 ,

For the case (ii), we see that $T=\left(T^{\prime} \cup U\right)+\left(\bigcup_{i=1}^{n} h_{i}\right)$ is ribbon-move equivalent to $T^{\prime}$. We consider two movies for a classical knot diagram $D$ in a plane, one of which keeps $D$ still and the other twists $D$ by a $2 \pi$-rotation of the plane. Then it follows from the definitions [2, 5, 9, that the corresponding Khovanov-Jacobsson homomorphisms $H^{*}(D) \rightarrow H^{*}(D)$ are the same for these movies. This implies that $T^{\prime}$ has the same Khovanov-Jacobsson number as that of a non-turned (that is, just spun) $\mathbb{T}^{2}$-knot, which is ribbon. Hence this case reduces to (i).

\section{ACKNOWLEDGEMENT}

The third author expresses his gratitude for the hospitality of the University of South Florida and the University of South Alabama. The first author thanks the organizers of Knots in Washington, May 2004; this conference stimulated our interest in the subject.

\section{REFERENCES}

1. S. Asami and S. Satoh, An infinite family of non-invertible surfaces in 4-space, Bull. London Math. Soc. 37 (2005), 285-296. MR2119028

2. D. Bar-Natan, Khovanov's homology for tangles and cobordisms, preprint available at: http://arxiv.org/pdf/math.GT/0410495

3. J. Boyle, The turned torus knot in $S^{4}$, J. Knot Theory Ramifications 2 (1993), 239-249. MR 1238874 (94i:57037)

4. J.S. Carter and M. Saito, Knotted surfaces and their diagrams, Mathematical Surveys and Monographs, vol. 55, American Mathematical Society, Providence, RI, 1998. MR.1487374 (98m:57027)

5. M. Jacobsson, An invariant of link cobordisms from Khovanov's homology theory, Algebr. Geom. Topol. 4 (2004), 1211-1251 MR2113903

6. T. Kanenobu and A. Shima, Two filtrations of ribbon 2-knots, Topology Appl. 121 (2002), 143-168. MR1903688(2003h:57034)

7. A. Kawauchi, On pseudo-ribbon surface-links, J. Knot Theory Ramifications 11 (2002), 10431062. MR.1941684 (2003h:57035)

8. M. Khovanov, A categorification of the Jones polynomial, Duke Math. J. 101(3) (1999), 359-426. MR1740682 (2002j:57025)

9. - An invariant of tangle cobordisms, preprint available at: http://xxx.lanl.gov/ abs/math.GT/0207264

10. E. Ogasa, Ribbon-moves of 2-knots: the Farber-Levine pairing and the AtiyahPatodi-Sinder-Casson-Gordon-Ruberman $\widetilde{\eta}$-invariants of 2-knots, preprint available at: http://xxx.lanl.gov/abs/math.GT/0004007

11. S. Satoh, Surface diagrams of twist-spun 2-knots, J. Knot Theory Ramifications 11 (2002), 413-430. MR1905695 (2003e:57041)

12. MR 2140603

13. A. Shima, On simply knotted tori in $S^{4} I I$, Knots '96 (Tokyo), 551-568, World Sci. Publishing, River Edge, NJ, 1997. MR:1664987 (99m:57022)

14. M. Teragaito, Symmetry-spun tori in the four-sphere, Knots 90 (Osaka, 1990), 163-171, de Gruyter, Berlin, 1992. MR,1177421 (93g:57029) 
15. T. Yajima, On simply knotted spheres in $R^{4}$, Osaka J. Math. 1 (1964), 133-152. MR0172280 (30:2500)

16. T. Yashiro, Deformations of surface diagrams, talk at First KOOK Seminar International Knot Theory and Related Topics, July 2004.

Department of Mathematics, University of South Alabama, Mobile, Alabama 36688

E-mail address: carter@jaguar1.usouthal.edu

Department of Mathematics, University of South Florida, Tampa, Florida 33620

E-mail address: saito@math.usf.edu

Graduate School of Science and Technology, Chiba University, Yayoi-cho 1-33, InAGE-KU, ChibA, 263-8522, JAPAN

E-mail address: satoh@math.s.chiba-u.ac.jp 